\title{
Identification of high-copy number long terminal repeat retrotransposons and their expansion in Phalaenopsis orchids
}

\author{
Chia-Chi Hsu', Shu-Yun Chen ${ }^{1}$, Pei-Han Lai ${ }^{1}$, Yu-Yun Hsiao ${ }^{2}$, Wen-Chieh Tsai ${ }^{3}$, Zhong-Jian Liư ${ }^{4}$, Mei-Chu Chung ${ }^{5}$, \\ Olivier Panaud ${ }^{6}$ and Hong-Hwa Chen ${ }^{1,2^{*}}$
}

\begin{abstract}
Background: Transposable elements (TEs) are fragments of DNA that can insert into new chromosomal locations. They represent a great proportion of eukaryotic genomes. The identification and characterization of TEs facilitates understanding the transpositional activity of TEs with their effects on the orchid genome structure.

Results: We combined the draft whole-genome sequences of Phalaenopsis equestris with BAC end sequences, Roche 454, and Illumina/Solexa, and identified long terminal repeat (LTR) retrotransposons in these genome sequences by using LTRfinder and classified by using Gepard software. Among the 10 families Gypsy-like retrotransposons, three families Gypsy1, Gypsy2, and Gypsy3, contained the most copies among these predicted elements. In addition, six high-copy retrotransposons were identified according to their reads in the sequenced raw data. The 12-kb Orchid-rt1 contains 18,000 copies representing $220 \mathrm{Mbp}$ of the P. equestris genome. Southern blot and slot blot assays showed that these four retrotransposons Gypsy1, Gypsy2, Gypsy3, and Orchid-rt1 contained high copies in the large-genome-size/large-chromosome species P. violacea and P. bellina. Both Orchid-rt1 and Gypsy1 displayed various ratios of copy number for the LTR sequences versus coding sequences among four Phalaenopsis species, including $P$. violacea and $P$. bellina and small-genome-size/small-chromosome $P$. equestris and $P$. ahprodite subsp. formosana, which suggests that Orchid-rt1 and Gypsy1 have been through various mutations and homologous recombination events. FISH results showed amplification of Orchid-rt1 in the euchromatin regions among the four Phalaenopsis species. The expression levels of Peg018599 encoding copper transporter 1 is highly upregulated with the insertion of Orchid-rt1, while it is down regulated for Peq009948 and Peg014239 encoding for a $26 S$ proteasome non-ATP regulatory subunit 4 homolog and auxin-responsive factor AUX/IAA-related. In addition, insertion of Orchid-rt1 in these three genes are all in their intron regions.
\end{abstract}

Conclusion: Orchid-rt1 and Gypsy1-3 have amplified within Phalaenopsis orchids concomitant with the expanded genome sizes, and Orchid-rt1 and Gypsy1 may have gone through various mutations and homologous recombination events. Insertion of Orchid-rt1 is in the introns and affects gene expression levels.

Keywords: Expression level, Genome size, Gypsy, Orchid-rt1, Intron, Orchids, Phalaenopsis, Retrotransposon

\footnotetext{
* Correspondence: hhchen@mail.ncku.edu.tw

'Department of Life Sciences, National Cheng Kung University, Tainan,

Taiwan

${ }^{2}$ Orchid Research and Development Center, National Cheng Kung University,

Tainan, Taiwan

Full list of author information is available at the end of the article
}

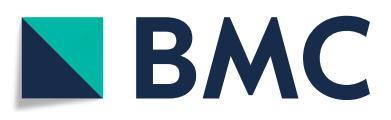

(- The Author(s). 2020 Open Access This article is licensed under a Creative Commons Attribution 4.0 International License, which permits use, sharing, adaptation, distribution and reproduction in any medium or format, as long as you give appropriate credit to the original author(s) and the source, provide a link to the Creative Commons licence, and indicate if changes were made. The images or other third party material in this article are included in the article's Creative Commons licence, unless indicated otherwise in a credit line to the material. If material is not included in the article's Creative Commons licence and your intended use is not permitted by statutory regulation or exceeds the permitted use, you will need to obtain permission directly from the copyright holder. To view a copy of this licence, visit http://creativecommons.org/licenses/by/4.0/. The Creative Commons Public Domain Dedication waiver (http://creativecommons.org/publicdomain/zero/1.0/) applies to the data made available in this article, unless otherwise stated in a credit line to the data. 


\section{Background}

Containing more than 25,000 species, Orchidaceae family, classified in class Liliopsida, order Asparagales, is one of the largest angiosperm families. The genus Phalaenopsis, an elegant and popular orchid, comprises approximately 63 species [1]. All diploid species of Phalaenopsis have the same chromosome number $(2 \mathrm{n}=$ $2 x=38$ ) [1], but their DNA contents and karyotypes vary significantly. The $\mathrm{C}$ value refers to the nuclear DNA contents of the replicated haploid genome and represents the genome size of species [2]. Flow cytometry revealed that the $2 \mathrm{C}$ values range from $2.77 \mathrm{pg}$ for $P$. philippinensis to $17.47 \mathrm{pg}$ for $P$. lobbii within the 50 species of Phalaenopsis [3, 4]. Two native species in Taiwan, $P$. equestris and $P$. aphrodite subsp. formosana (hereafter called $P$. aphrodite), have relatively small genome sizes, with $2 \mathrm{C}$ values of 2.95 and $2.81 \mathrm{pg}$, equivalent to $1.6-$ and 1.52-Gbp genome sequences [3, 4]. However, the scented Phalaenopsis species $P$. violacea and P. bellina have a large genome (Additional file 1: Fig. S1), with $2 \mathrm{C}$ values of 12.89 and $13.04 \mathrm{pg}$, respectively, equivalent to 6.99- and 7.07-Gbp genome sizes.

Karyotype analysis of nine Phalaenopsis species revealed the genome size associated with the chromosome size. One group with small and uniform chromosomes (1-2.5 $\mu \mathrm{m}$ ) includes $P$. aphrodite, $P$. stuartiana, $P$. equestris, $P$. cornu-cervi and $P$. lueddemanniana, and the other with bimodal karyotypes, with small, medium and large chromosomes, includes $P$. venosa, $P$. amboinensis, $P$. violacea and $P$. mannii [5]. Different chromosome sizes among various species result in incompatability for interspecific crosses [5-7], thus limiting the breeding of new varieties for the orchid industry. The amount of constitutive heterochromatin in the interphase nucleus indicates that Phalaenopsis species with large chromosomes contain more heterochromatin and repetitive sequences than those with small chromosomes [5].

Mobile or transposable elements (TEs) are DNA fragments that can "jump" to new chromosomal locations and thus often duplicate themselves. They represent a large part of eukaryotic genomes, ranging from 3\% in baker's yeast (Saccharomyces cerevisiae) [8], 20\% in fruit fly (Drosophila melanogaster) [9], 45\% in humans (Homo sapiens) [10], $50 \%$ in grape (Vitis vinifera), 59\% in Sorghum (Sorghum bicolor), 60\% in Amborella (Amborella trichopoda), $62 \%$ in P. equestris, and $75 \%$ in maize (Zea mays) [11]. In general, there are two major groups of TEs distinguished by their transposition intermediate: class I retrotransposons with "copy-and-paste" retrotransposition, and class II DNA transposons with "cut-and-paste" retrotransposition [12].

Retrotransposons with the "copy-and-paste" mechanism represent the largest portion of higher plant genomes, and long terminal repeat (LTR) retrotransposons are the most common and ubiquitous in the plant kingdom [13, 14]. LTR retrotransposons are related to retroviruses in terms of their structure and retrotransposition mechanism. The complete copies of retrotransposons consist of two LTRs that flank an internal region containing two genes, gag and pol. The gag gene encodes a capsid-like protein, and the pol gene encodes a polyprotein with protease, reverse transcriptase, RNase $\mathrm{H}$, and integrase activities. LTR retrotransposons are further classified into two major families, Ty1/copia-like and Ty3/gypsy-like elements, depending on the order of reverse transcriptase and integrase.

LTR sequences are responsible for transcription initiation and termination of the internal genes for retrotransposition, leading to an increase in copy number. In contrast, the increased genome size can be mitigated by unequal homologous recombination, generating a much shorter solo LTR, or by illegitimate recombination, leading to element deletion [15]. Therefore, differential efficiency of such increasing versus decreasing mechanisms among plant genomes could explain at least in part the various genome sizes in recently described plant species [15].

Although LTR retrotransposons comprise the largest fraction of most plant genomes, only a few LTR retrotransposon families successfully represent the bulk of the genome. The main TE groups are ancient and are present in all plant kingdoms, yet TE contents show extreme diversity among species in the classes of TEs present, their fraction in the genome and level of transposition activity. Identifying TEs and investigating their impact on each plant species are essential for understanding their functional roles. However, precise characterization of TEs is not easy or straightforward. First, many TEs are structurally incomplete because of intra- or inter-element unequal recombination or accumulation of small deletions by illegitimate recombination $[16,17]$. Second, many TEs are grouped in nested patterns [18] or chimerical structures [19]. Finally, lowcopy-number TE families are highly divergent across species, and their identification is difficult by sequence homology because of few numbers of previously characterized elements. Hence, the full characterization of TEs is a critical step for precise gene annotation in a sequenced genome and investigating the effects between TEs and genome evolution.

Although the whole-genome sequences of Phalaenopsis have been published for two native Taiwan species, $P$. equestris and $P$. aphrodite $[11,20]$, few studies have focused on the TEs contributing to their genome structure and evolution. Recently, a $P$ instability factor-like transposon, PePIF1, was isolated from P. equestris and seemed to be actively transposed during microprogation [21]. A retrotransposon, Harlequin Orchid RetroTransposon 1 (HORT1), is inserted in the upstream regulatory sequence 
of an anthocyanin-related PeMYB11 and results in its high expression and the harlequin/black flowers of Phalaenopsis orchids [22].

In this study, we have identified four high-copy retrotransposons, and further examined in both smallgenome-size/small-chromosome Phalaenopsis species ( $P$. aphrodite and $P$. equestris) and large-genome-size/largechromosome species (P. bellina and P. violacea).

\section{Results}

\section{Identification of long terminal repeat (LTR)} retrotransposons

The LTR retrotransposons were identified from several sequenced genome datasets for Phalaenopsis orchids, including BAC end sequences (BESs) [23], Roche 454, Illumina/Solexa, and assembled draft whole genome sequences [11], by using two approaches. First, these sequences were assembled into large contigs, then LTRfinder was used to identify the full-length LTR retrotransposons, which are bordered by two identical LTRs at the time of insertion and subsequently diverged by random mutations [24]. Second, the high-copy numbers of retrotransposons with high similarities were predicted from the sequencing reads, then those with the highest copies in the sequenced raw data were selected.

In total, 906 Gypsy-like and 402 Copia-like LTR retrotransposons were identified as the full-length elements by using LTRfinder, then further separated into different families according to their sequence homology by using a Dot-Plot software, Gepard [25]. Overall, 906 Gypsy-like retrotransposons were grouped into 10 families (Table 1), and 402 Copia-like retrotransposons were distributed into 20 families (Additional file 2: Table S1). Three Gypsy-like families, Gypsy1-Gypsy3, contained the most full-length copies among these predicted elements. The Gypsy1 family contained 13,824-bp sequences with 1722-bp LTR sequences and a 5110-bp protein-coding region (Table 1, Fig. 1). The Gypsy2 family had 13,646bp sequences with 1720-bp LTR sequences and a 5106bp protein-coding region (Table 1, Fig. 1). The Gypsy3 family had 13,889-bp sequences with 1641-bp LTR sequences and a 5084-bp protein-coding region (Table 1, Fig. 1). The length of the full element of Gypsy4 10 ranged from 8663to 16,646-bp sequences with 1023- to 2642-bp sequences of LTR sizes. In contrast, the Copia-like retrotransposons contained lower copy numbers than those of Gypsy, with the highest copy number of 48 in 402 elements (Additional file 2: Table S1).

Considering that the new amplified retrotransposons with very similar sequence would be assembled into the same contig after next-generation sequencing and could not be identified as a high-copy element by LTRfinder and Gepard, we used a second approach of high-copy retrotransposons with high similarity predicted as the most sequenced reads in the sequenced raw data. In total, six high-copy number retrotransposons were identified, including one $16 \mathrm{~S}$ ribosomal DNA (Orchidits16S), one miniature inverted-repeat TE (mite)-type retrotransposon (Orchid-mite1), one anc-like retrotransposon (Orchid-rt-anc1), and three retrotransposons (Orchid-rt1, Orchid-rt2, and Orchid-rt3) (Table 2). Among them, Orchid-rt1 is a Gypsy-like retrotransposon and contains 12,671-bp sequences with 707-bp LTR sequences and a 5121-bp protein-coding region (Fig. 1). Based on the numbers of these sequenced reads, Orchid$r t 1$ is predicted to be present with 18,000 copies in the $P$. equestris genome, accounting for $220-\mathrm{Mbp}$ sequences and $13.8 \%$ of the Phalaenopsis genome.

\section{Slot blot assay of high copy number of retrotransposons present in Phalaenopsis genomes}

Because the repetitive sequences represent a large proportion of the plant genomes and play roles in genomic diversification and speciation [26-28], a rapid burst of

Table 1 The copy numbers of the predicted Gypsy-like retrotransposons

\begin{tabular}{lllll}
\hline Family name & No. of elements & Total length (bp) & LTR length & CDS length (bp) \\
\hline Gypsy1 & 234 & 13,824 & 1722 & 5110 \\
Gypsy2 & 201 & 13,646 & 1720 & 5106 \\
Gypsy3 & 185 & 13,889 & 1641 & 5084 \\
Gypsy4 & 47 & 13,180 & 1629 & 3356 \\
Gypsy5 & 39 & 14,447 & 1872 & 5099 \\
Gypsy6 & 28 & 15,199 & 1949 & 4880 \\
Gypsy7 & 20 & 15,648 & 1813 & 3145 \\
Gypsy8 & 13 & 8663 & 1023 & 3346 \\
Gypsy9 & 11 & 16,646 & 2642 & 3453 \\
Gypsy10 & 3 & 15,195 & 1526 & 3212 \\
Singleton & 125 & & \\
total & 906 & & & \\
\hline
\end{tabular}




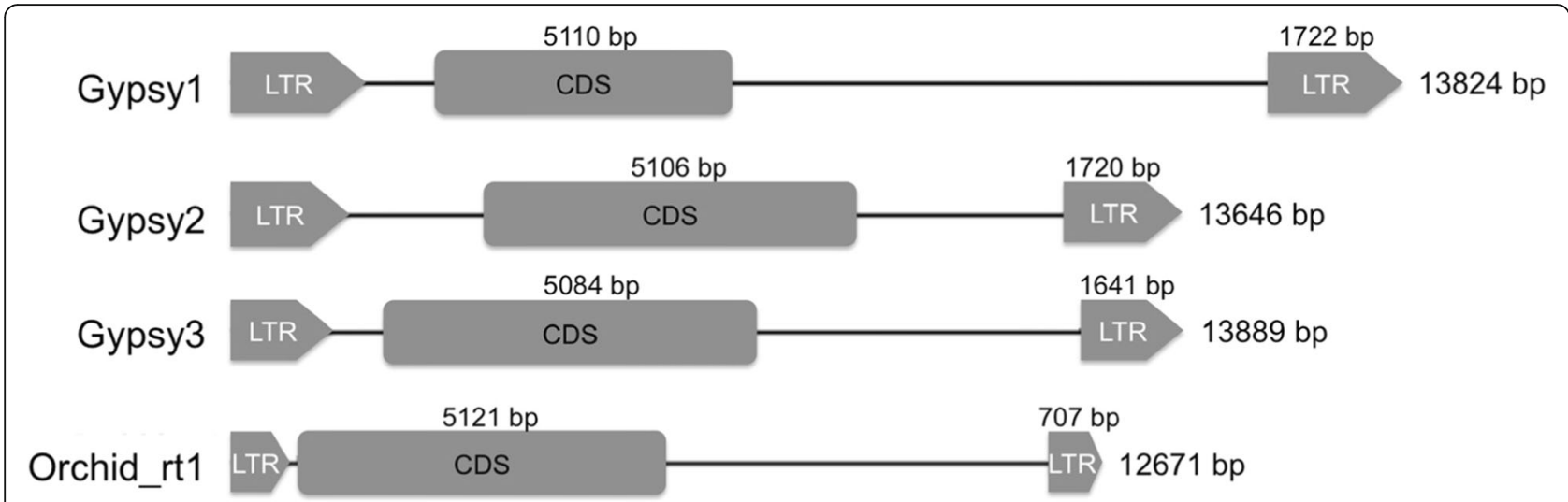

Fig. 1 A diagram for the sequence structures of the Gypsy1 to Gypsy3 and Orchid-rt1. Long terminal repeats (LTRs) and protein-coding sequence (CDS) were shown as arrows and boxes, respectively

retrotransposition could induce genome size changes with different evolutionary effects in the same genus, as reported for Oryza, Nicotiana, and Genlisea [29]. To trace the retrotransposition events of the four high-copy LTR retrotransposons (Gypsy1, Gypsy2, Gypsy3, and Orchid-rt1) in four Phalaenopsis species, slot blot analysis was used to estimate the copy number of these highcopy retrotransposons with the probes corresponding to the individual LTR region. Four Phalaenopsis genomes were analyzed including $P$. equestris and $P$. aphrodite with small chromosomes, and $P$. violacea and $P$. bellina with both large and small chromosomes [5].

A 10-ng plasmid DNA, containing a 707-bp LTR sequence of Orchid-rt1 and a 3015-bp sequence of T-easy vector, was used as a standard containing the Orchidrt1-LTR sequence for $1.9 \mathrm{ng}$ (Fig. 2). The slot blot results showed that $3^{-2} \mathrm{x}$ diluted 10-ng Orchid-rt1-LTR plasmid DNA revealed a similar intensity of hybridized spots as $3^{-1} \mathrm{x}$ diluted genomic DNA of P. equestris (Fig. 2a, arrowheads), which suggests an approximately 0.63-ng (1.9 ng $\div 3)$ LTR sequence for Orchid-rt1 within $1 \mu \mathrm{g}$ genomic DNA of $P$. equestris. With the genome size of $P$. equestris of $1.6 \times 10^{9} \mathrm{bp}$ [11], the LTR sequence for Orchid-rt1 was $1.01 \times 10^{6} \mathrm{bp}$, for about 1426 copies within the $P$. equestris genome (Table 3). The genome sizes of $P$. equestris, $P$. aphrodite, P. violacea, and P. bellina were compared to their nuclear DNA content (Table 3); the estimated copy numbers of LTR sequences for Orchid-rt1 were 1426, 4085, 18,785, and 19,000 in the genomes of $P$. equestris, $P$. aphrodite, P. violacea, and $P$. bellina, respectively (Table 3 ).

Similarly, a 10-ng plasmid DNA, having a 5121-bp protein-coding sequence (CDS) of Orchid-rt1 and a 3015-bp sequence of T-easy vector, was used as a standard with the Orchid-rt1-CDS of $6.3 \mathrm{ng}$ (Fig. 2). Slot blot results showed that $3^{-3} \mathrm{x}$ diluted 10-ng Orchid-rt1-CDS plasmid DNA revealed a similar intensity of hybridized spots as $3^{-3} \mathrm{x}$ diluted genomic DNA of $P$. equestris (Fig. 2b, arrowheads), which suggests an approximate 6.3-ng CDS for Orchid-rt1 within $1 \mu$ g genomic DNA of P. equestris. So the CDS for Orchid-rt1 was $10.08 \times 10^{6}$ $\mathrm{bp}$, for about 1968 copies in the $P$. equestris genome (Table 3), and the estimated copy numbers of the CDS for Orchid-rt1 were 1870, 8599, and 8698 within the genomes of $P$. aphrodite, $P$. violacea, and $P$. bellina, respectively (Table 3 ).

In comparing the copy numbers of LTR and CDS for Orchid-rt1, we found nearly 2-fold copy numbers of

Table 2 The copy numbers of the predicted high-copy orchid retrotransposons

\begin{tabular}{llllllll}
\hline element & size & $\mathbf{4 5 4}$ & BES & Illimina & \% 454 & \% BES & \% Illumina \\
\hline Orchid-its165 & 2060 & 1625 & 345 & 20,914 & 0.004 & 0.019 & 0.006 \\
Orchid-mite1 & 317 & 500 & 30 & 664 & 0.001 & 0.002 & 0.000 \\
Orchid-rt-anc1 & 3396 & 6961 & 764 & 59,493 & 0.016 & 0.041 & 0.018 \\
Orchid-rt1 & 12,754 & 57,245 & 2927 & 358,553 & 0.130 & 0.158 & 0.107 \\
Orchid-rt2 & 3059 & 4650 & 247 & 41,109 & 0.011 & 0.013 & 0.012 \\
Orchid-rt3 & 3410 & 17,457 & 1999 & 112,842 & 0.040 & 0.108 & 0.034 \\
Total & & 439,604 & 18,486 & $3,354,752$ & & & \\
\hline
\end{tabular}




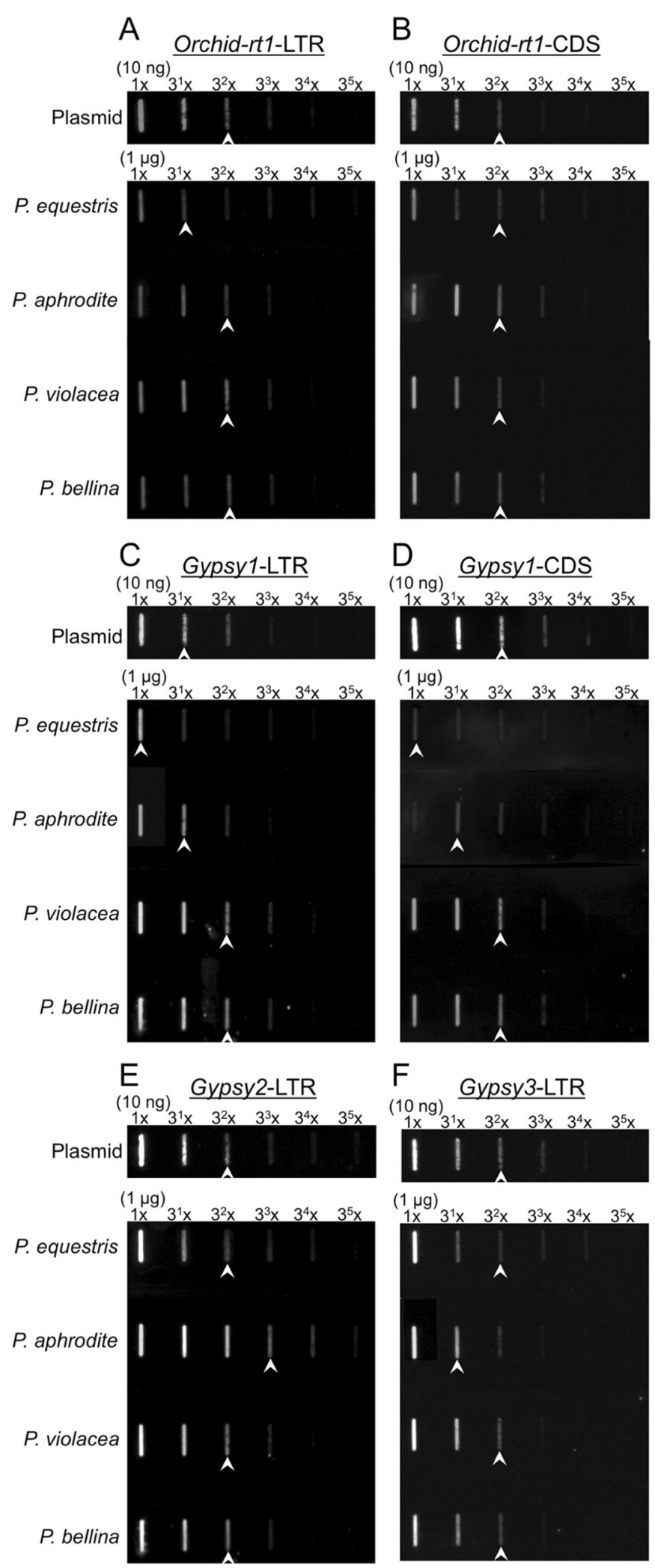

Fig. 2 (See legend on next page.) 
(See figure on previous page.)

Fig. 2 Slot Blot showed the percentage of the LTR $(\mathbf{a}, \mathbf{c})$ and $\operatorname{CDS}(\mathbf{b}, \mathbf{d})$ sequences of Orchid-rt1 $(\mathbf{a}, \mathbf{b})$ and Gypsy1 (c, d), and the LTR sequences of Gypsy2 (e) and Gypsy3 (f) within the genomic DNA of P. equestris, P. aphrodite, P. violacea, and P. belllina. A 10 ng of plasmid DNA containing the LTR sequences and $1 \mu \mathrm{g}$ genomic DNA were performed for serial dilution and hybridized with the probes of PeAct9 and these LTR sequences. Arrowheads indicate the similar intensity of hybridized slots between the plasmid DNA and genomic DNA

LTR versus CDS in the genomes of $P$. aphrodite, $P$. violacea, and $P$. bellina, so most of Orchid-rt1 involved full-length copies flanked by two LTR sequences in these genomes. However, P. equestris showed lower copies of LTR than CDS for Orchid-rt1, with 1426 and 1968 copies, respectively (Table 3 ). In the "increase/decrease model", whereby following the retrotransposon amplification, these sequences without selection pressure are eliminated by homologous or illegitimate recombination and lead to the solo-LTR formation and deletion [15], the copies of Orchid-rt1 in P. equestris were amplified a long time ago and underwent faster TE turnover rate than that in P. aphrodite, P. violacea, and P. bellina.

Increased copy number of Gypsy1 coincident with largegenome-size/large-chromosome Phalaenopsis species The copy numbers of LTR and CDS for Gypsy1 and the LTR for Gypsy 2 and Gypsy3 were estimated in these four Phalaenopsis genomes (Table 3). All retrotransposons contained high copy numbers in the large-chromosome $P$. violacea and $P$. bellina genomes (Table 3 ), with a much higher copy number of LTR for Gypsy1 in both $P$. violacea and $P$. bellina genomes, 43,840 and 44,341 copies, respectively (Table 3 ). Considering that the genome sizes were about 4.5 -fold higher for $P$. violacea and $P$. bellina than $P$. equestris and $P$. aphrodite, the copy numbers of LTR and CDS for Gypsy1 showed about a 39- and 13-fold increase in both $P$. violacea and $P$. bellina as compared with $P$. equestris and $P$. aphrodite, respectively (Table 3), which suggests that Gypsy1 may be linked to the increased chromosome size as well as genome size in $P$. violacea and P. bellina. In addition, Gypsy1 showed about 5 -fold more copies of LTR than CDS in all four Phalaenopsis genomes (Table 3), which indicates that Gypsy1 amplified its copies a long time ago and each copy underwent deletion and recombination events, which resulted in solo-LTR copies [15].

Even though both $P$. equestris and $P$. ahprodite have similar genome size, we found a prevalence for Gypsy 2 or Gypsy3 in the P. aphrodite or P. equestris genome. The LTR copy number for Gypsy 2 was higher in $P$. aphrodite than $P$. equestris, but that for Gypsy3 was higher in $P$. equestris than $P$. aphrodite (Table 3). Therefore, the evolutionary fates among the four retrotransposons varied among Phalaenopsis genomes and should be investigated separately.

\section{Chromosomal localization of Orchid-rt1 in Phalaenopsis genomes}

Orchid-rt1, accounting for $13.8 \%$ of the P. equestris genome size was the highest-copy retrotranspsons and with high similarity among copies. We analyzed its distribution patterns on chromosomes of P. equestris, P. aphrodite, $P$. violacea, and $P$. bellina by using fluorescence in

Table 3 Estimated copies of four transposable elements among the genomes of four Phalaenopsis species

\begin{tabular}{|c|c|c|c|c|c|}
\hline & & P. equestris & P. aphrodite & P. violacea & P. bellina \\
\hline \multicolumn{2}{|c|}{ Nuclear DNA content $1 \mathrm{C}$ value $(\mathrm{pg})$} & 2.95 & 2.81 & 12.89 & 13.04 \\
\hline \multicolumn{2}{|c|}{ Estimated genome size ( $\left.10^{9} \mathrm{bp}\right)$} & 1.6 & 1.52 & 6.99 & 7.07 \\
\hline \multirow[t]{4}{*}{ 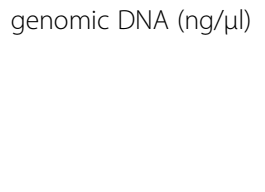 } & Orchid-rt1-LTR/CDS & $0.63 / 6.3$ & $1.9 / 6.3$ & $1.9 / 6.3$ & $1.9 / 6.3$ \\
\hline & Gypsy1-LTR/CDS & $1.2 / 0.7$ & $3.6 / 2.1$ & $10.8 / 6.3$ & $10.8 / 6.3$ \\
\hline & Gypsy2-LTR & 3.6 & 10.8 & 3.6 & 3.6 \\
\hline & Gypsy3-LTR & 3.5 & 1.17 & 3.5 & 3.5 \\
\hline \multirow[t]{4}{*}{ Mb/genome } & Orchid-rt1-LTR/CDS & $1.008 / 10.08$ & $2.888 / 9.576$ & $13.281 / 44.037$ & $13.433 / 44.541$ \\
\hline & Gypsy1-LTR/CDS & $1.92 / 1.12$ & $5.472 / 3.192$ & 75.492/44.037 & $76.356 / 44.541$ \\
\hline & Gypsy2-LTR & 5.76 & 16.416 & 25.164 & 25.452 \\
\hline & Gypsy3-LTR & 5.6 & 1.778 & 24.465 & 24.745 \\
\hline \multirow[t]{4}{*}{ Copy no. /genome } & Orchid-rt1-LTR/CDS & $1426 / 1968$ & $4085 / 1870$ & $18,785 / 8599$ & $19,000 / 8698$ \\
\hline & Gypsy7-LTR/CDS & $1115 / 219$ & $3178 / 625$ & $43,840 / 8618$ & $44,341 / 8716$ \\
\hline & Gypsyz-LTR & 3349 & 9544 & 14,630 & 14,798 \\
\hline & Gypsy3-LTR & 3413 & 1083 & 14,909 & 15,079 \\
\hline
\end{tabular}


situ hybridization (FISH). Both P. equestris and P. aphrodite have small and uniform chromosomes, whereas $P$. bellina and $P$. violace have bimodal karyotypes with small, medium and large chromosomes (Fig. 3). FISH results showed Orchid-rt1 dispersed through all chromosomes in P. equestris (Fig. 3a) and P. aphrodite (Fig. 3c). Because the intensity of FISH signals may reflect the abundance of sequences clustered at the regions, $P$. bellina (Fig. $3 e$ ) and $P$. violacea (Fig. 3g) have more Orchid-rt1 as compared with $P$. equestris (Fig. 3a) and P. aphrodite (Fig. 3c). In P. bellina (Fig. 3e) and P. violacea (Fig. 3g), Orchid-rt1 repeats distributed in the intercalary regions of medium and large chromosomes and throughout all small chromosomes. According to the intensity of 4', 6-diamidino-2-phenylindole (DAPI) staining, the intercalary regions of the medium and large chromosomes were mainly euchromatin, flanked by conspicuous heterochromatin (Fig. 3f, h).

\section{Gene expression affected by Orchid-rt1 insertion}

It has been found that the $P$. equestris genome has high abundance (about 60\%) of transposon elements (TEs). Unlike other plants, these TEs are enriched in the intron regions [11]. Recently, we found that gene expression of PeMYB11 is highly upregulated by the insertion of a retrotransposon, HORT1 and results dark flower color in harlequin Phalaenopsis [22]. We wondered whether the insertion of Orchid-rt1 may affect gene expressions. For this, whole genome alignment was performed by using the full length of Orchid-rt1 against the P. equestris genome sequence. Among them, 38 of 43 hits were located on chromosomes, and 5 hits were located on unassembled scaffolds. In addition, 7 of 38 hits were inserted within genes, thus their paralogous genes were also identified. We then compared the gene expression levels among various organs of the 7 genes inserted with $\mathrm{Or}$ chid-rt1 and their paralogs from the RNAseq data in OrchidBase (http://orchidbase.itps.ncku.edu.tw).

To confirm whether these genes do contain portions of Orchid-rt1 sequence, primers around the predicted Orchid-rt1 insertion sites were designed from three predicted Orchid-rt1 inserted genes, Peq018599, Peq009948, and Peq014239. The amplified regions were sequenced and aligned to the Orchid-rt1 sequence. A 600-bp fragment from Orchid-rt1 was found within the predicted insertion region from Peq009948 (Additional file 3: Fig. S2), but only short and separated matched fragments for the other two genes. This suggests that some predicted gene do harbor the Orchid-rt1 region.

Among these 7 genes, expression levels of one gene (Peq018599) is highly upregulated in all organs as compared to that of its paralogous genes, while expression levels of 2 genes (Peq009948 and Peq014239) are down-

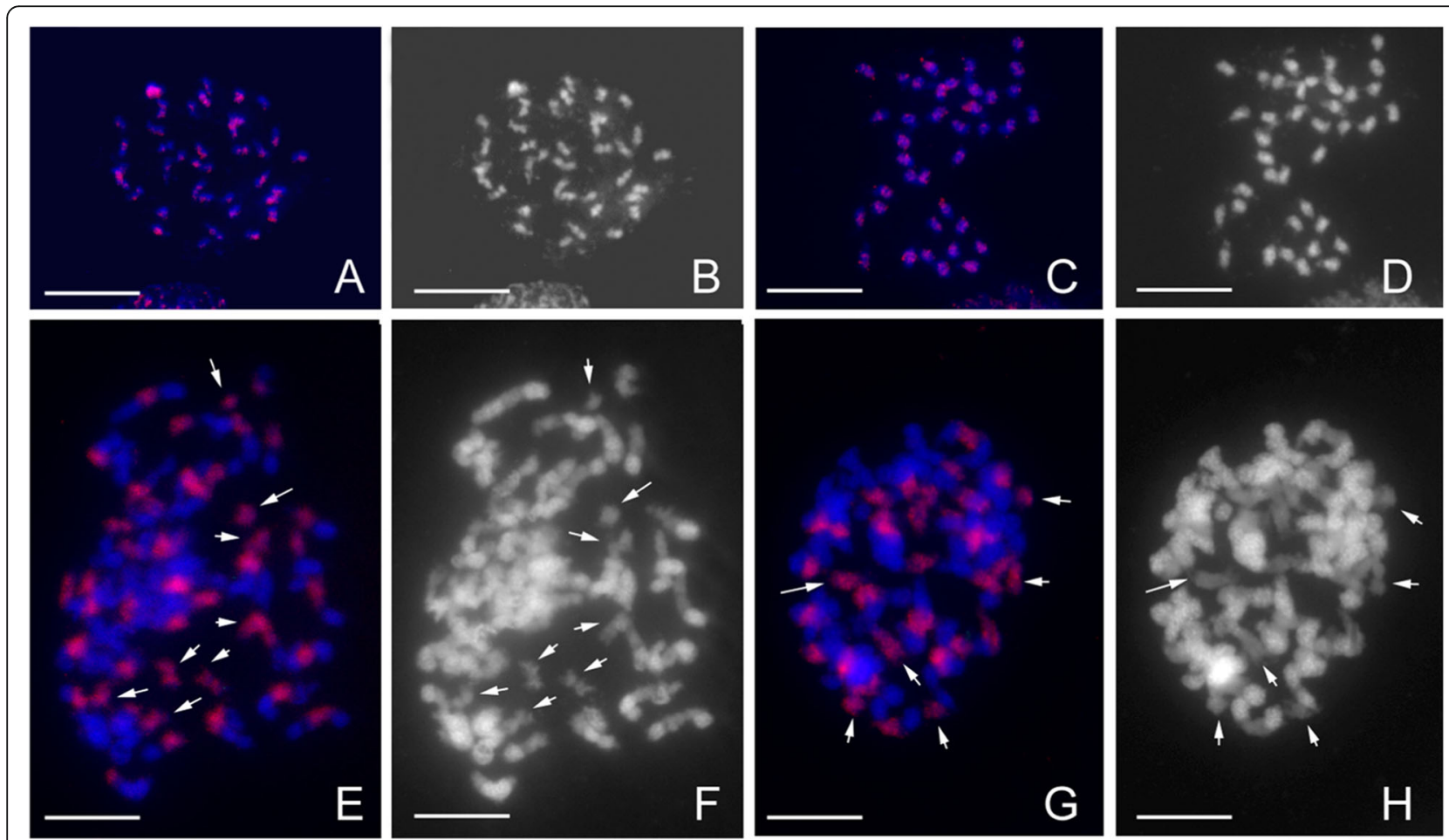

Fig. 3 Fluorescence in situ hybridization (FISH) mapped the LTR sequence of Orchid-rt1 on chromosomes of $P$. equestris (a-b), $P$. aphrodite subsp. formosana (c-d), P. bellina (e-f), and P. violacea ( $\mathbf{g}-\mathbf{h})$. $\mathbf{a}, \mathbf{c}$, e and $\mathbf{g}$ The distribution of Orchid-rt1 (red) on chromosomes (blue). $\mathbf{b}, \mathbf{d}, \mathbf{f}, \mathbf{h}$ The respective images of chromosomes with DAPI staining. Arrows indicate the small chromosomes in $P$. bellina and $P$. violacea 
regulated as compared to that of their paralogous genes (Fig. 4a). qRT-PCR was applied to validate the expression level of the predicted Orchid-rt1 inserted genes and its paralogous genes (Fig. 4b). The predicted Orchid-rt1 inserted gene Peq018599 showed up-regulated expression as compared to its paralogous genes Peq025947 and Peq023950 in RNA-seq data and confirmed with qRTPCR. In contrast, the other two predicted Orchid-rt1 inserted genes Peq009948 and Peq014239 showed downregulated expression compared to their paralogous genes Peq019291 and Peq000381, respectively, in RNA-seq analysis, and this was confirmed by using qRT-PCR (Fig. $4 \mathrm{~b})$. This indicates that there might be association between the TE insertion and gene expression.

Expression levels of four other genes were not significantly affected with the insertion of Orchid-rt1 as compared to their paralogs (Additional file 4: Table S2).
Intriguingly, the above three genes (Peq018599, Peq009948, and Peq014239) with their expression levels been traumatized all with the insertion of Orchid-rt1 in the intron regions with various size of $765 \mathrm{bp}, 473 \mathrm{bp}$ and $297 \mathrm{bp}$, respectively (Fig. 5).

\section{Discussion}

In this study, we identified several LTR retrotransposons from $P$. equestris genome sequences for full-length and high-copy elements and characterized their amount and distribution pattern in four Phalaenopsis genomes. Among them, four Gypsy-like LTR retrotransposons, Orchid-rt1, Gypsy1, Gypsy2, and Gypsy3, represented a large amount of the Phalaenopsis genomes, while the increased copies of both Orchid-rt1 and Gypsy1 in two species with large genome size/chromosome size, $P$. violacea and $P$. bellina, suggest that these two

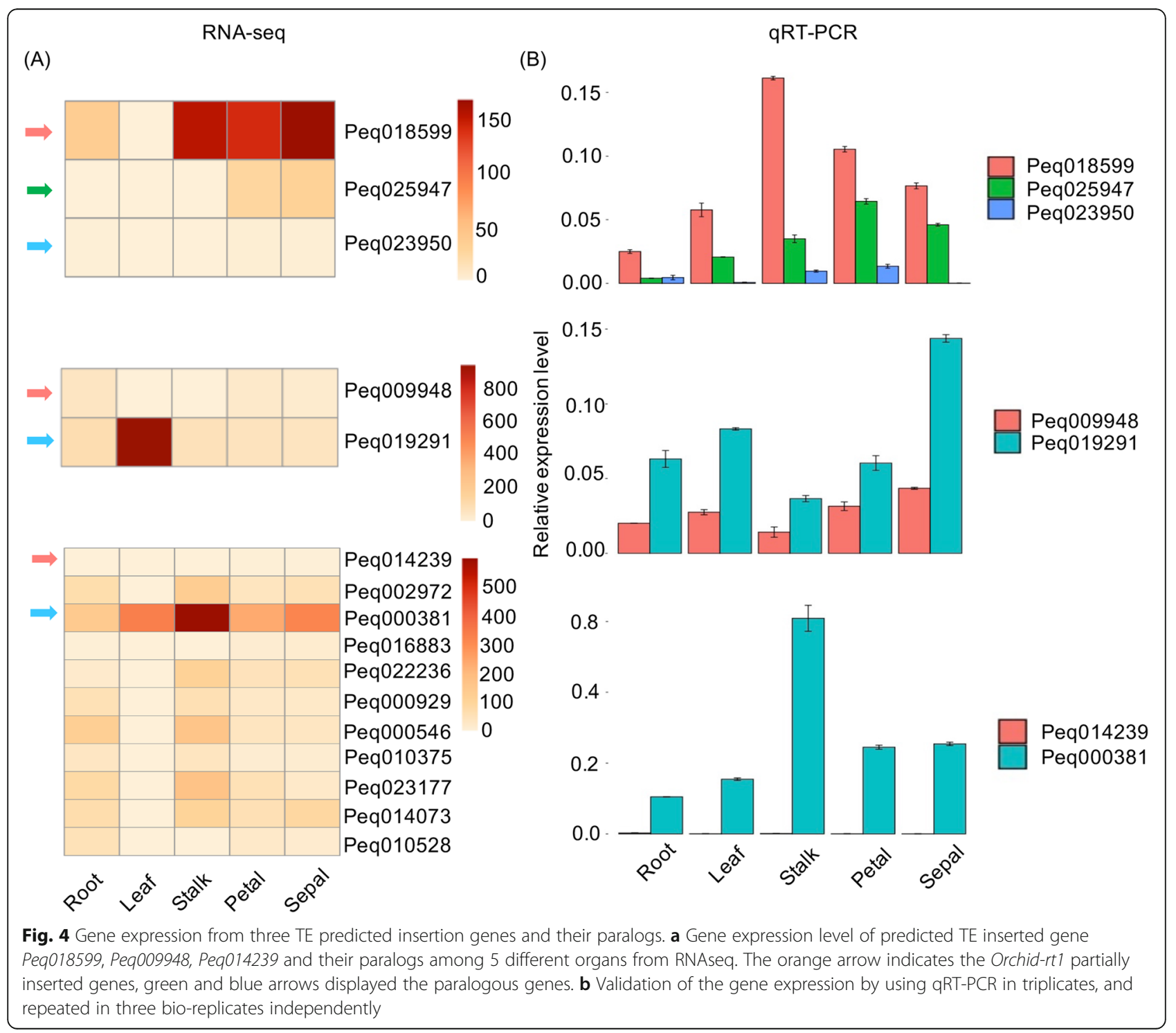




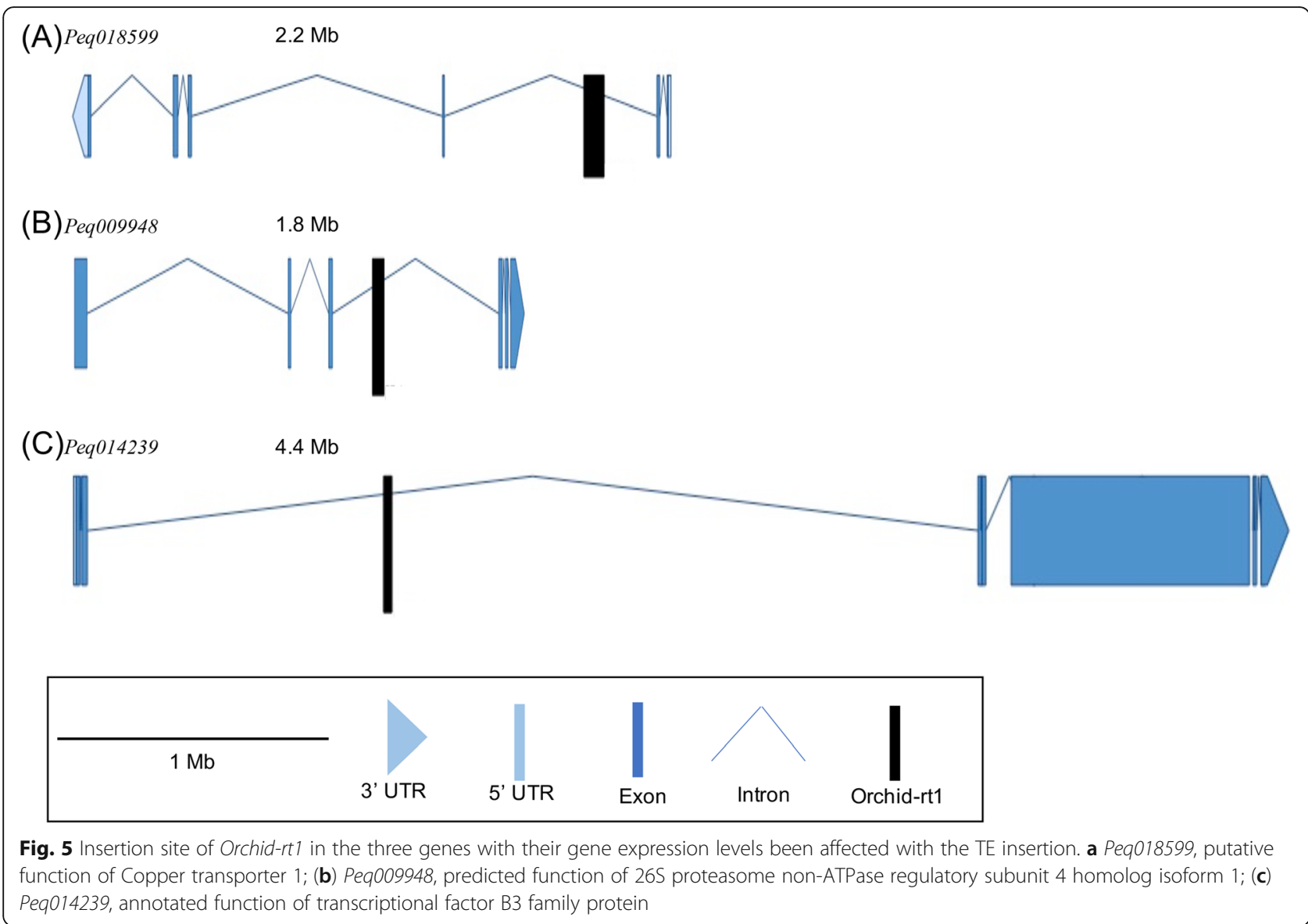

retrotransposons are associated with genome size expansion. Based on a big comparative study for eight highquality genomes, including Arabidopsis thaliana, A. lyrata, Brachypodium dystachion, grapevine (Vitis vinifera), maize (Zea mays), rice (Oryza sativa), sorghum (Sorghum bicolor), and soybean (Glycine max), it is a clear correlation between genome size to the retrotranspositional activity of the few retrotransposon families with most highly repeated, but not to a high number of repeated families [30]. Moreover, only one or few LTR retrotransposons were active recently in each genome, and the latest retrotranspositional burst(s) mostly were accounted for the difference in plant genome sizes [30]. Therefore, it is necessary to identify the LTR retrotransposons and investigate their effects on each plant genome.

Two approaches have been performed for the identification of the high-copy LTR retrotransposons from the Phalaenopsis genome, including LTRfinder for the fulllength LTR retrotransposons from the assembled sequences, and the prediction of high-copy retrotransposons with high similarity from the most sequenced reads in the sequenced raw data. The prediction of LTRfinder for the full-length LTR retrotransposons with the protein-coding region for retrotransposition bordered by two identical
LTRs was performed in several plant genomes, such as cassava (Manihot esculenta) [31], citrus (Citrus $x$ clementina) [32], cotton (Gossypium hirsutum) [33], flax (Linum usitatissimum L.) [34], Medicago truncatula [35], and wheat (Triticum aestivum) [36]. Therefore, the 906 Gypsylike and 402 Copia-like retrotransposons identified in this study were the predicted full-length retrotransposons with the protein-coding region bordered by two identical LTRs.

In addition, considering the composition of LTR retrotransposons is originated from the copy-and-paste strategy, the reads from the sequenced raw data will be assembled into a single contig among the draft genome sequence based on the sequence similarity. To avoid this, the second approach was performed via the prediction of high-copy retrotransposons with high similarities from the most sequenced reads in the sequenced raw data. Therefore, Orchid-rt1 has been identified and predicted to be present with 18,000 copies in the P. equestris genome, but only 43 hits could be identified from the assembled whole genome sequences. The fact of high-copy numbers of Orchid-rt1 present in Phalaenopsis genomes was then confirmed by using Southern blot, slot blot, and FISH results. It indicated that each copy of Orchid-rt1 was similar to each other, while the amplified 
copies of Orchid-rt1 were recently occurred without many mutations accumulated. As a fate of the retrotransposition event for LTR retrotransposons, the sequences of the newly inserted copy are identical to the original one, while mutations accumulated and resulting sequence divergence of these two copies over time, whose extent is proportional to the time elapsed since the insertion [15].

FISH results showed Orchid-rt1 dispersed throughout all chromosomes in P. equestris and P. aphrodite, while only in the intercalary regions of medium and large chromosomes and throughout all small chromosomes in $P$. bellina and $P$. violacea. Considering that the studies of genome size variation among Phalaenopsis species show a positive relation between genome size and the amount of constitutive heterochromatin, the differential accumulation of heterochromatin is considered a major cause of karyotype variation in Phalaenopsis orchids [5]. Therefore, FISH results showed amplification of Orchid$r t 1$ in the euchromatin regions also contributing to the expanded genome sizes. Similar situation was also found in the cytogenetic analysis on Brachiaria genus, while an Athila retrotransposon was detected mainly in the centromeric-pericentromeric regions of chromosomes of diploids $B$. decumbens, B. ruziziensis, and B. brizantha, but differences in location and signal strength in the polyploid B. brizantha [37].

Expression levels of genes inserted with Orchid-rt1 in $P$. equestris genome are upregulated, downregulated, or non-significantly affected. We found 3 genes that have their expression levels regulated with the Orchid-rt1 insertion. Peq018599 encoding for copper transporter 1 with the insertion of Orchid-rt1 showed highly upregulated gene expression in root and seed development as compared to its paralogs. Copper transporter 1 plays an important role in symbiotic nitrogen fixation in Medicago truncatula [38], functions in root elongation and pollen development in Arabidopsis [39], and involves in the copper transport in root of grapevine [40]. Two genes, Peq009948 and Peq014239 encoding for 26S proteasome non-ATPase regulatory subunit 4 homolog isoform 1 and transcriptional factor B3 family protein/ auxin-responsive factor AUX/IAA-related, respectively, were down-regulated in all organs and seed development as compared to their paralogs. The $26 \mathrm{~S}$ proteasome is a complex in charge of protein turn over. The $26 \mathrm{~S}$ proteasome contains one $20 \mathrm{~S}$ core particle and two $19 \mathrm{~S}$ regulatory particles (RPs). The RPN12a protein, a nonATPase subunit of the 19S RP, is involved in cytokinin signaling in Arabidopsis [41]. Expression level of Peq009948 is reduced to half amount of its paralog in most organs, and abolished in the floral stalk and leaf. Auxin response factor is a member of the plant-specific B3 DNA binding superfamily [42]. In the Peq014239, its expression is almost abolished in the floral stalk, leaf and root, trace amount in sepal and seed development, and a substantial amount in petal, labellum, pollinium and gynostemium. All these three genes are involved in growth and development, and further analysis will be required to detail the significance of regulation of gene expression upon the insertion of Orchid-rt1 into these genes.

\section{Conclusion}

In this study, we identified several LTR retrotransposons from $P$. equestris genome sequences for full-length and high-copy elements and characterized their amount and distribution pattern in four Phalaenopsis genomes. Four Gypsy-like LTR retrotransposons, Orchid-rt1, Gypsy1, Gypsy2, and Gypsy3, represented a large amount of the Phalaenopsis genomes. Both Orchid-rt1 and Gypsy1 showed increased copies in two species with large genome size/chromosome size, $P$. violacea and $P$. bellina, which suggests that these two retrotransposons may be associated with genome size expansion. FISH results revealed that abundant Orchid-rt1 mainly distributed in the intercalary euchromatin regions of large chromosomes in these two species. Our results support that these four retrotransposons amplified themselves within Phalaenopsis genomes, accompanied by expanded genome sizes. Insertion of Orchid-rt1 is found in the intron regions and gene expression are affected upon the insertion of Orchid-rt1.

\section{Methods}

\section{Plant materials}

We tested four native Phalaenopsis orchids with small genome size/small chromosomes or large genome size/ large chromosomes accompanied by small chromosomes. $P$. aphrodite and $P$. equestris have small genomes, and $P$. bellina and $P$. violacea have large genomes. All plant materials were grown in the greenhouse at National Cheng Kung University (Tainan, Taiwan) under natural light and controlled temperature from $23^{\circ} \mathrm{C}$ to $27^{\circ} \mathrm{C}$.

\section{Sequencing the Phalaenopsis genomic DNA}

The analyzed sequences were obtained from the previously sequenced 21,350 BAC end sequences (BESs), and the sequenced Phalaenopsis genome by using an NGS strategy with the Illumina/Solexa platform. The genomic DNA was extracted from leaves of $P$. equestris, sheared into 40-kb sequences by using HydroShear (Digilab, Marlborough, MA, USA), and sequenced by using Roche 454 and Illumina/Solexa for one run. The sequences from the BESs, Roche 454, and Illumina/Solexa data were assembled into large contigs by using Sequencher 4.2 (Gene Codes Corp., Ann Arbor, MI). 


\section{Identification and characterization of TEs in $P$. equestris genome}

The assembled large contigs were used to search for TEs with on-line TE-prediction software, LTRfinder, LTRharvest, and RepeatMasker. LTRfinder and LTRharvest are software tools for automated annotation of internal features of putative LTR retrotransposons. RepeatMasker is used to screen DNA sequences for interspersed repeats and low-complexity DNA sequences. Each predicted TE was verified for its terminal repeats on both ends and identified for other copies in the Phalaenopsis genome by using NCBI-BLAST2 Sequences (http://www.ncbi.nlm. nih.gov/ blast/bl2seq/wblast2.cgi/) and Gepard, a rapid and sensitive tool for creating dotplots on a genome scale [25]. NCBI-BLAST2 Sequences and Gepard provide easy and powerful means of sequence analysis, useful for searching repeats within a single sequence and regions of similarity in two sequences. The TEs with their similar copies were grouped into the same families. The probable protein-coding regions among the terminal repeat sequences were predicted by using BLASTX with the NCBI non-redundant (nr) protein sequences database. The length of LTR sequences for each predicted LTR retrotransposon were analyzed by using NCBI-BLAST2 Sequences and multiple alignment software. The autonomous elements and the complete one for each TE family were retained for finding the other copies for each TE by using BLASTN. Each TE family was identified and characterized with its sequence structure and copy number in the Phalaenopsis genome. Moreover, each characterized TE was analyzed for their contribution to Phalaenopsis genome structure.

\section{Slot blot hybridization}

Genomic DNAs were isolated from flowers of P. aphrodite, $P$. equestris, $P$. bellina and $P$. violacea. Slot blot assay was performed with $1 \mu \mathrm{g}$ genomic DNA, $10 \mathrm{ng}$ plasmid DNA, and a serial dilution of DNAs. These DNAs were loaded onto nylon filters by using Hoefer PR648 (Hoefer Inc., Holliston, MA, USA). Probes were amplified from the genomic DNA of $P$. equestris for the LTR sequences for Orchid-rt1, Gypsy1, Gypsy2, and Gypsy3 and the open reading frame sequences for Orchid-rt1 and Gypsy1. Slot blot hybridization followed the standard protocol [43] with the AlkPhos Direct Labelling and Detection System and the CDP-Star kit (Amersham Pharmacia Biotech).

\section{Fluorescence in situ hybridization (FISH) analysis}

Preparation of $P$. equestris, $P$. aphrodite, $P$. violacea, and $P$. bellina chromosome spreads and FISH procedures followed dual-color FISH protocols [44]. Briefly, root tips were treated with $2 \mathrm{mM}$ 8-hyroxyquinoline, fixed with Farmer's solution, and macerated with $2 \%$ cellulose
(Onozuka R-10, Yakult Honsha, Tokyo) and 2\% pectinase (Sigma Chemical Co.) in $10 \mathrm{mM}$ citrate buffer, $\mathrm{pH}$ 4.0 , at $37^{\circ} \mathrm{C}$ for $90 \mathrm{~min}$, then squashed on slides in the same fixative. The probe for the LTR sequence for Orchid-rt1 was labeled with digoxigenin-11-dUTP (Roche Diagnostics) via nick translation according to a standard protocol. Post-hybridization washing, signal detection, and image processing were performed as described [44]. Chromosomes were counterstained with 4', 6diamidino-2-phenylindole (DAPI) in an antifade solution (Vector Laboratories, CA, USA). FISH images were recorded by use of an epifluorescence microscope (AxioImager A1, Carl Zeiss AG, Jena, Germany) equipped with a cooled CCD camera (AxioCam MRm). Figures were edited by using Adobe Photoshop 9.0 (Adobe Systems Inc., USA). For each accession, at least five chromosome complements with good labelling signals were photographed and analyzed.

\section{Expression levels of genes inserted by Orchid-rt1 and their paralogs}

To find out the genes that have insertions of Orchid-rt1, whole genome alignment was performed by using the full length of Orchid-rt1 against the P. equestris genome sequence with the software LAST [45]. Among them, 38 of 43 hits were located on chromosomes, and the others were located on unassembled scaffolds. Furthermore, 7 of 38 were participated within the genes. The paralog genes of above 7 genes were identified by using the full CDS for tblastx in Orchidbase database (http://orchidbase.itps. ncku.edu.tw/ est/home2012.aspx) based on the threshold that score higher than 100 and $\mathrm{E}$ value lower than 0.00001. To confirm the insertion of Orchid-rt1 in the predicted genes, primers were design from outside of predicted insertion sites by primer3plus (http://www.bioinformatics.nl/cgi-bin/primer3plus/ primer3plus.cgi/). The amplified fragments were sequenced and BLAST against Orchid-rt1 full-length sequence using the global alignment in NCBI (https://blast.ncbi.nlm.nih.gov/Blast.cgi).

The relative gene expression levels of those genes inserted with Orchid-rt1 and their paralogous genes in several organs were found in the Orchidbase as well and presented as heatmap. The up-regulated or downregulated gene expression of the inserted genes compared to their paralogs identified in the RAN-seq data were further confirmed by using qRT-PCR. The cDNA samples were prepared from 5 different organs including root, leaf, stalk, petal and sepal. Were mixed with $2 \mathrm{X}$ SYBR Green PCR master mix (Applied Biosystems, Norwalk, CT, USA) and analyzed in an ABI 7300 instrument (Applied Biosystems). Each sample was performed in triplicates as technical repeats with the following reactions: Incubation at $50{ }^{\circ} \mathrm{C}$ for $2 \mathrm{~min}$, then $95^{\circ} \mathrm{C}$ for 10 min, and thermal cycling for 40 cycles $\left(95^{\circ} \mathrm{C}\right.$ for $15 \mathrm{~s}$ and 
$60{ }^{\circ} \mathrm{C}$ for $\left.1 \mathrm{~min}\right)$. The relative expression level was calculated according to the manufacturer's instructions (Applied Biosystems). qRT-PCR was performed with three different plants as bio-replicates. The Peactin1 was applied as internal control for normalization.

\section{Supplementary Information}

The online version contains supplementary material available at https://doi. org/10.1186/s12864-020-07221-6.

Additional file 1: Figure S1. Four native Phalaenopsis species analyzed in the study, including $P$. aphrodite subsp. formosana (A) and $P$. equestris (B) with small genome/small chromosomes, and $P$. bellina $(C)$ and $P$. violacea (D) with large genome/large chromosomes.

Additional file 2: Table S1. The copy numbers of the predicted Copialike retrotransposons.

Additional file 3: Figure S2. The global alignment result between Orchid-rt1 full length and predicted insertion region from Pea009948.

Additional file 4: Table S2. The expression levels of Orchid-rt1 inserted gene and their paralogs genes among different organs.

\section{Abbreviations}

BES: BAC end sequences; CDS: Coding sequence; FISH: Fluorescence in situ hybridization; LTRs: Long Terminal Repeats; RPs: Regulatory particles; TEs: Transposable elements

\section{Acknowledgments}

This work was supported by the Ministry of Science and Technology, Taiwan (MOST) (grant no. MOST 106-2811-B-006-057-).

\section{Availability of supporting data}

The data sets supporting the results of this article are included within the article (Additional files 1).

\section{Authors' contributions}

$\mathrm{CCH}, \mathrm{SYC}, \mathrm{PHL}$, and MCC performed the experiments, wrote and corrected the manuscript. CCH and OP performed bioinformatic analysis. YYH, WCT, and ZJL provided the Phalaenopsis genome sequences. CCH, SYC, OP, and $\mathrm{HHC}$ designed the study, checked the data analyses, and organized, wrote and corrected the manuscript. All authors have read and approved the manuscript.

\section{Funding}

This work was supported by the Ministry of Science and Technology, Taiwan (MOST) (grant no. MOST 106-2811-B-006-057-).

\section{Availability of data and materials}

Original sequences of the BESs, Roche 454, Illumina/Solexa data, and the identified LTR retrotransposons can be accessed at Orchidbase database (http://orchidbase.itps.ncku.edu.tw/est/home2012.aspx).

\section{Ethics approval and consent to participate}

Not applicable.

\section{Consent for publication}

Not applicable.

\section{Competing interests}

The authors declare that they have no competing interests.

\section{Author details}

'Department of Life Sciences, National Cheng Kung University, Tainan, Taiwan. ${ }^{2}$ Orchid Research and Development Center, National Cheng Kung University, Tainan, Taiwan. ${ }^{3}$ Institute of Tropical Plant Sciences and Microbiology, National Cheng Kung University, Tainan, Taiwan. ${ }^{4}$ Key Laboratory of National Forestry and Grassland Administration for Orchid Conservation and Utilization at College of Landscape Architecture, Fujian
Agriculture and Forestry University, Fuzhou, China. ${ }^{5}$ Institute of Plant and Microbial Biology, Academia Sinica, Nankang, Taipei, Taiwan. ${ }^{6}$ Institute of Plant Genome and Development, University of Perpignan, Perpignan, France.

Received: 22 May 2020 Accepted: 9 November 2020

Published online: 19 November 2020

\section{References}

1. Christenson EA. Phalaenopsis. Portland: Timber Press; 2001.

2. Bennetzen JL. Transposable elements, gene creation and genome rearrangement in flowering plants. Curr Opin Genet Dev. 2005:15:621-7.

3. Lin S, Lee HC, Chen WH, Chen CC, Kao YY, Fu YM, et al. Nuclear DNA contents of Phalaenopsis species and Doritis pulcherrima. J Amer Soc Hort Sci. 2001;126:195-9.

4. Chen WH, Kao YK, Tang CY, Tsai CC, Lin TY. Estimating nuclear DNA content within 50 species of the genus Phalaesnopsis Blume (Orchidaceae). Sci Hortic. 2013;161:70-5.

5. Kao YY, Chang SB, Lin TY, Hsieh CH, Chen YH, Chen WH, et al. Differential accumulation of heterochromatin as a cause of karyotype variation in Phalaenopsis orchids. Ann Bot. 2001:87:387-95.

6. Shinodo K, Kamemoto H. Karyotype analysis of some species of Phalaenopsis. Cytologia. 1963;28:390-8.

7. Arends JC. Cytological observations on genome homology in eight interspeific hybrids of Phalesnopsis. Genetica. 1970:41:88-100.

8. Kim JM, Vanguri S, Boeke JD, Gabriel A, Voytas DF. Transposable elements and genome organization: a comprehensive survey of retrotransposons revealed by the complete Saccharomyces cerevisiae genome sequence. Genome Res. 1998:8:464-78.

9. Kapitonov W, Jurka J. Molecular paleontology of transposable elements in the Drosophila melanogaster genome. Proc Natl Acad Sci U S A. 2003;100: 6569-74.

10. The International Human Genome Sequencing Consortium (TIHGSC). Initial sequencing and analysis of the human genome. Nature. 2001:409:860-921.

11. Cai J, Liu X, Vanneste K, Proost S, Tsai WC, Liu KW, et al. The genome sequence of the orchid Phalaenopsis equestris. Nat Genet. 2015:47:65-72.

12. Finnegan DJ. Eukaryotic transposable elements and genome evolution. Trends Genet. 1989;5:103-7.

13. Kumar A, Bennetzen JL. Plant retrotransposons. Annu Rev Genet. 1999;33: 479-532.

14. Meyers BC, Tingey SV, Morgante M. Abundance, distribution, and transcriptional activity of repetitive elements in the maize genome. Genome Res. 2001:11:1660-76.

15. Vitte C, Panaud O. LTR-retrotransposons and flowering plant genome size: emergence of the increase/decrease model. Cytogenet Genome Res. 2005; 110:91-107.

16. Devos KM, Brown JK, Bennetzen JL. Genome size reduction through illegitimate recombination counteracts genome expansion in Arabidopsis. Genome Res. 2002;12:1075-9.

17. Ma J, Devos KM, Bennetzen JL. Analyses of LTR-retrotransposon structures reveal recent and rapid genomic DNA loss in rice. Genome Res. 2004;14: 860-9.

18. SanMiguel P, Tikhonov A, Jin YK, Motchoulskaia N, Zakharov D, MelakeBerhan A, et al. Nested retrotransposons in the intergenic regions of the maize genome. Science. 1996;274:765-8.

19. Jiang N, Bao Z, Zhang X, Eddy SR, Wessler SR. Pack-MULE transposable elements mediate gene evolution in plants. Nature. 2004;431:569-73.

20. Chao YT, Chen WC, Chen CY, Ho HY, Yeh CH, Kuo YT, et al. Chromosomelevel assembly, genetic and physical mapping of Phalaenopsis aphrodite genome provides new insights into species adaptation and resources for orchid breeding. Plant Biotechnol J. 2018;16:2027-41.

21. Hsu CC, Lai PH, Chen TC, Tsai WC, Hsu JL, Hsiao YY, et al. PePIF1, a P-lineage of PIF-like transposable element identified in protocorm-like bodies of Phalaenopsis orchids. BMC Genomics. 2019:20:25.

22. Hsu CC, Su CJ, Jeng MF, Chen WH, Chen HH. A HORT1 retrotransposon insertion in the PeMYB11 promoter causes harlequin/black flowers in Phalaenopsis orchids. Plant Physiol. 2019:180:1535-48.

23. Hsu CC, Chung YL, Chen TC, Lee YL, Kuo YT, Tsai WC, et al. An overview of the Phalaenopsis orchid genome through BAC end sequence analysis. BMC Plant Biol. 2011;11:3

24. Xu Z, Wang H. LTR_FINDER: an efficient tool for the prediction of full-length LTR retrotransposons. Nucleic Acids Res. 2007;35:W265-8. 
25. Krumsiek J, Arnold R, Ratter T. Gepard: a rapid and sensitive tool for creating dotplots on gneome scale. Bioinformatics. 2007;23:1026-8.

26. Neumann P, Koblizkova A, Navratilova A, Macas J. Significant expansion of Vicia pannonica genome size mediated by amplification of a single type of giant retroelement. Genetics. 2006;173:1047-56.

27. Hawkins JS, Proulx SR, Rapp RA, Wendel JF. Rapid DNA loss as a counterbalance to genome expansion through retrotransposon proliferation in plants. Proc Natl Acad Sci U S A. 2009;106:17811-6.

28. Gill N, SanMiguel P, Dhillon BDS, Abernathy B, Kim HR, Stein L, et al. Dynamic Oryza genomes: repetitive DNA sequences as genome modeling agents. Rice. 2010;3:251-69.

29. Levy AA. In: Fedoroff NV, editor. Transposons in plant speciation: John Wiley \& Sons, Inc; 2013.

30. Baidouri ME, Panaud O. Comparative genomic paleontology across plant kingdom reveals the dynamics of TE-driven genome evolution. Genome Biol Evol. 2013;5:954-65.

31. Oliveira-Silva AM, Silva GF, Dias MC, Clement CR, Sousa NR. Interretrotransposon-amplified polymorphism markers for germplasm characterization in Manihot esculenta (Euphorbiaceae). Genet Mol Res. 2014; 13:3800-4.

32. Du D, Du X, Mattia MR, Wang Y, Yu O, Huang M, et al. LTR retrotransposons from the Citrus $x$ clementina genome: characterization and application. Tree Genet Genomes. 2018;14:43.

33. Wang K, Zhang W, Cao Y, Zhang Z, Zheng D, Zhou B, et al. Localization of high level of sequence conservation and divergence regions in cotton. Theor Appl Genet. 2012;124:1173-82.

34. González LG, Deyholos MK. Identification, characterization and distribution of transposable elements in the flax (Linum usitatissimum L.) genome. BMC Genomics. 2012;13:644.

35. Wang H, Liu JS. LTR retrotransposon landscape in Medicago truncatula: more rapid removal than in rice. BMC Genomics. 2008;9:382.

36. Garbus I, Romero JR, Valarik M, Vanžurová H, Karafiátová M, Cáccamo M, et al. Characterization of repetitive DNA landscape in wheat homeologous group 4 chromosomes. BMC Genomics. 2015;16:375.

37. Santos FC, Guyot R, do Valle CB, Chiari L, Techio VH, Heslop-Harrison P, et al. Chromosomal distribution and evolution of abundant retrotransposons in plants: gypsy elements in diploid and polyploid Brachiaria forage grasses. Chromosom Res. 2015;23:571-82.

38. Senovilla M, Castro-Rodríguez R, Abreu I, Escudero V, Kryvoruchko I, Udvardi MK, et al. Medicago truncatula copper transporter 1 (MtCOPT1) delivers copper for symbiotic nitrogen fixation. New Phytol. 2018;218:696-709.

39. Sancenón V, Puig S, Mateu-Andrés I, Dorcey E, Thiele DJ, Peñarrubia L. The Arabidopsis copper transporter COPT1 functions in root elongation and pollen development. J Biol Chem. 2004;279:15348-55.

40. Martins V, Bassil E, Hanana M, Blumwald E, Gerós H. Copper homeostasis in grapevine: functional characterization of the Vitis vinifera copper transporter 1. Planta. 2014;240:91-101.

41. Ryu MY, Cho SK, Kim WT. RNAi suppression of RPN12a decreases the expression of type-a ARRS, negative regulators of cytokinin signaling pathway, in Arabidopsis. Mol Cell. 2009;28:375-82.

42. Wen J, Guo P, Ke Y, Liu M, Li P, Wu Y, et al. The auxin response factor gene family in allopolyploid Brassica napus. PLoS One. 2019;14:e0214885.

43. Sambrook J, Fritsch EF, Maniatis T. Molecular cloning: a laboratory manual. 3rd ed. Cold Spring Harbor: Cold Spring Harbor Laboratory Press; 2001.

44. Chung MC. Chromosome techniques and FISH. In: Yeung ECT, et al., editors. Plant microtechniques and protocols: Springer International Publishing Switzerland; 2015. p. 287-309. https://doi.org/10.1007/978-3-319-19944-3_17.

45. Kielbasa SM, Wan R, Sato K, Horton P, Frith MC. Adaptive seeds tame genomic sequence comparison. Genome Res. 2011;21:487-93.

\section{Publisher's Note}

Springer Nature remains neutral with regard to jurisdictional claims in published maps and institutional affiliations.

Ready to submit your research? Choose BMC and benefit from:

- fast, convenient online submission

- thorough peer review by experienced researchers in your field

- rapid publication on acceptance

- support for research data, including large and complex data types

- gold Open Access which fosters wider collaboration and increased citations

- maximum visibility for your research: over $100 \mathrm{M}$ website views per year

At BMC, research is always in progress.

Learn more biomedcentral.com/submissions 